\title{
Optimal-transport formulation of electronic density-functional theory
}

\author{
Giuseppe Buttazzo, ${ }^{1}$ Luigi De Pascale, ${ }^{2}$ and Paola Gori-Giorgi ${ }^{3}$ \\ ${ }^{1}$ Dipartimento di Matematica, Università di Pisa, Largo B. Pontecorvo 5 - 56127 Pisa, Italy \\ ${ }^{2}$ Dipartimento di Matematica Applicata, Università di Pisa, Via Buonarroti 1/C - 56127 Pisa, Italy \\ ${ }^{3}$ Department of Theoretical Chemistry, Amsterdam Center for Multiscale Modeling, FEW, Vrije Universiteit, \\ De Boelelaan 1083, 1081HV Amsterdam, The Netherlands
}

(Received 25 September 2011; revised manuscript received 11 January 2012; published 1 June 2012)

\begin{abstract}
The most challenging scenario for Kohn-Sham density-functional theory, that is, when the electrons move relatively slowly trying to avoid each other as much as possible because of their repulsion (strong-interaction limit), is reformulated here as an optimal transport (or mass transportation theory) problem, a well-established field of mathematics and economics. In practice, we show that to solve the problem of finding the minimum possible internal repulsion energy for $N$ electrons in a given density $\rho(\mathbf{r})$ is equivalent to find the optimal way of transporting $N-1$ times the density $\rho$ into itself, with the cost function given by the Coulomb repulsion. We use this link to set the strong-interaction limit of density-functional theory on firm ground and to discuss the potential practical aspects of this reformulation.
\end{abstract}

DOI: 10.1103/PhysRevA.85.062502

PACS number(s): 31.15.ec, 71.15.Mb, 02.90.+p

\section{INTRODUCTION}

Electronic structure theory plays a fundamental role in many different fields: material science, chemistry and biochemistry, solid-state physics, and surface physics. Its goal is to solve, in a reliable and computationally affordable way, the many-electron problem, a complex combination of quantum-mechanical and many-body effects. The most widely used technique, which achieves a reasonable compromise between accuracy and computational cost, is Kohn-Sham (KS) density-functional theory (DFT) [1,2].

Optimal transport or mass transportation theory studies the optimal transfer of masses from one location to another. Mass transportation theory dates back to 1781 when Monge [3] posed the problem of finding the most economical way of moving soil from one area to another, and received a boost when Kantorovich, in 1942, generalized it to what is now known as, the Kantorovich dual problem [4]. Optimal transport problems appear in various areas of mathematics and economics.

In this paper, we show that one of the most challenging scenarios for KS DFT, that is, when the repulsion between the electrons largely dominates over their kinetic energies, can be reformulated as an optimal transport problem. As we see, the potential of this link between two different well-established research areas has both formal and practical aspects.

It is difficult to write a paper fully accessible to two different communities, such as mass transportation and electronic density-functional theory. In an effort toward this goal, we have chosen to use, for both the optimal transport and the DFT parts, the most commonly used notation in each case, translating from one to the other throughout the paper. The paper is organized as follows. We start in Sec. II with a review of the motivations to study the strong-interaction limit of DFT and the challenges that this limit poses. Right after, in Sec. III, we discuss the implications of our mass transportation theory reformulation of this limit for DFT, anticipating the results that are derived in the subsequent sections. This way, the first part of the paper is a self-contained presentation written with language that is entirely familiar to the density-functional theory community. The mass transportation theory problem is then introduced in Sec. IV and is used in Secs. V and VI to address the strong-interaction limit of DFT and to derive the results anticipated in Sec. III. Simple examples, mainly thought to illustrate the problem for the mass transportation theory community, are given in Sec. VII. This second part of the paper is, thus, mainly written in a language familiar to the optimal transport reader. The last section, Sec. VIII, is devoted to a final discussion of the connection between these two different research areas and to conclusions and perspectives. Finally, many of the technical details are given in the Appendices.

\section{STRONG INTERACTIONS IN DFT}

In the formulation of Hohenberg and Kohn (HK) [1], electronic ground-state properties are calculated by minimizing the energy functional $E[\rho]$ with respect to the particle density $\rho(\mathbf{r})$

$$
E[\rho]=F[\rho]+\int v_{\mathrm{ext}}(\mathbf{r}) \rho(\mathbf{r}) d \mathbf{r},
$$

where $v_{\text {ext }}(\mathbf{r})$ is the external potential and $F[\rho]$ is a universal functional of the density, defined as the expectation value of the internal energy (kinetic energy $\hat{T}=$ $-\frac{1}{2} \sum_{i=1}^{N} \nabla_{i}^{2}$ plus electron-electron interaction energy $\hat{V}_{e e}=$ $\sum_{i=1}^{N} \sum_{j=i+1}^{N}\left|\mathbf{r}_{i}-\mathbf{r}_{j}\right|^{-1}$ ) of the minimizing wave function that yields the density $\rho(\mathbf{r})$ [5],

$$
F[\rho]=\min _{\Psi \rightarrow \rho}\left\langle\Psi\left|\hat{T}+\hat{V}_{e e}\right| \Psi\right\rangle .
$$

Here and throughout the paper, we use Hartree atomic units $\left(\hbar=m_{e}=a_{0}=e=1\right)$.

In the standard Kohn-Sham approach [2], the minimization of $E[\rho]$ in Eq. (1) is performed under the assumption that the kinetic energy dominates over the electron-electron interaction by introducing the functional $T_{s}[\rho]$, corresponding to the minimum of the expectation value of $\hat{T}$ alone over all fermionic (spin- $\frac{1}{2}$ particles) wave functions yielding the given $\rho$ [5],

$$
T_{s}[\rho]=\min _{\Psi \rightarrow \rho}\langle\Psi|\hat{T}| \Psi\rangle .
$$


The functional $T_{s}[\rho]$ defines a noninteracting reference system with the same density as the interacting one. The remaining part of the exact energy functional,

$$
E_{\mathrm{Hxc}}[\rho] \equiv F[\rho]-T_{s}[\rho]
$$

is approximated. Usually, $E_{\mathrm{Hxc}}[\rho]$ is split as $E_{\mathrm{Hxc}}[\rho]=$ $U[\rho]+E_{x c}[\rho]$, where $U[\rho]$ is the classical Hartree functional,

$$
U[\rho]=\frac{1}{2} \int d \mathbf{r} \int d \mathbf{r}^{\prime} \frac{\rho(\mathbf{r}) \rho\left(\mathbf{r}^{\prime}\right)}{\left|\mathbf{r}-\mathbf{r}^{\prime}\right|},
$$

and the exchange-correlation energy $E_{x c}[\rho]$ is the crucial quantity that is approximated.

The KS approach works well in many scenarios, but as expected, runs into difficulty where particle-particle interactions play a more prominent role. In such cases, the physics of the HK functional $F[\rho]$ is completely different than the one of the Kohn-Sham noninteracting system so that trying to capture the difference $F[\rho]-T_{s}[\rho]$ with an approximate functional is a daunting task. A piece of exact information on $E_{x c}[\rho]$ is provided by the functional $V_{e e}^{\mathrm{SCE}}[\rho]$, defined as the minimum of the expectation value of $\hat{V}_{e e}$ alone over all wave functions yielding the given density $\rho(\mathbf{r})$,

$$
V_{e e}^{\mathrm{SCE}}[\rho]=\min _{\Psi \rightarrow \rho}\left\langle\Psi\left|\hat{V}_{e e}\right| \Psi\right\rangle .
$$

The acronym "SCE" stands for "strictly correlated electrons" [6]: $V_{e e}^{\mathrm{SCE}}[\rho]$ defines a system with maximum possible correlation between the relative electronic positions (in the density $\rho$ ), and it is the natural counterpart of the KS noninteracting kinetic energy $T_{s}[\rho]$. Its relevance for $E_{x c}[\rho]$ increases with the importance of particle-particle interactions with respect to the kinetic energy $[7,8]$. For low-density many-particle scenarios, it has been shown that $V_{e e}^{\mathrm{SCE}}[\rho]$ is a much better zero-order approximation to $F[\rho]$ than $T_{s}[\rho]$ [9-11]: This defines a "SCE-DFT" alternative and complementary to standard KS DFT. In more general cases, the dividing line between the regime where the KS approach with its current approximations works well and the regime where a SCE-based approach is more suitable is a subtle issue with many complex systems being not well described by either KS or SCE (see also the discussion in Ref. [11]).

The functional $V_{e e}^{\mathrm{SCE}}[\rho]$ also contains exact information on the important case of the stretching of the chemical bond $[11,12]$, a typical situation in which restricted KS DFT encounters severe problems. The relevance of $V_{e e}^{\mathrm{SCE}}[\rho]$ for constructing a new generation of approximate $E_{x c}[\rho]$ 's has also been pointed out very recently by Becke [13]. Notice that $V_{e e}^{\mathrm{SCE}}[\rho]$ also enters in the derivation of the Lieb-Oxford bound [14-18], an important exact condition on $E_{x c}[\rho]$.

Overall, constructing the functional $V_{e e}^{\mathrm{SCE}}[\rho]$ for a given density $\rho(\mathbf{r})$ in an exact and efficient way has the potential to extensively broaden the applicability of DFT. Only approximations for $V_{e e}^{\mathrm{SCE}}[\rho]$ were available [19] until recently, when the mathematical structure of the exact $V_{e e}^{\mathrm{SCE}}[\rho]$ had been investigated in a systematic way $[20,21]$ and exact solutions for spherically symmetric densities (which were used in the first SCE-DFT calculations [9,11]) were produced. However, a general reliable algorithm to construct $V_{e e}^{\mathrm{SCE}}[\rho]$ is still lacking, and many formal aspects still need to be addressed. Here is where mass transportation theory can play a crucial role.
Reformulating $V_{e e}^{\mathrm{SCE}}[\rho]$ as an optimal transport problem allows setting the construction of this functional on firm ground and importing algorithms from another well-established research field.

\section{RESULTS: AN OVERVIEW}

The problem posed by Eq. (6), that is, searching for the minimum possible interaction energy in a given density, was first addressed, in an approximate way, in the seminal papers of Seidl and co-workers [6,7,19]. Later on, in Refs. [20,21], a formal solution was given in the following way. The admissible configurations of $N$ electrons in $d$ dimensions are restricted to a $d$-dimensional subspace $\Omega_{0}$ of the full $N d$-dimensional configuration space. A generic point of $\Omega_{0}$ has the form $\mathbf{R}_{\Omega_{0}}(\mathbf{s})=\left(\mathbf{f}_{1}(\mathbf{s}), \ldots, \mathbf{f}_{N}(\mathbf{s})\right)$ where $\mathbf{s}$ is a $d$-dimensional vector that determines the position of, say, electron " 1 ," and $\mathbf{f}_{i}(\mathbf{s})$ $\left[i=1, \ldots, N, \mathbf{f}_{1}(\mathbf{s})=\mathbf{s}\right]$ are the co-motion functions, which determine the position of the $i$ th electron in terms of $\mathbf{s}$. The variable $\mathbf{s}$ itself is distributed according to the normalized density $\rho(\mathbf{s}) / N$. The comotion functions are implicit functionals of the density, determined by a set of differential equations that ensures the invariance of the density under the transformation $\mathbf{s} \rightarrow \mathbf{f}_{i}(\mathbf{s})$

$$
\rho\left(\mathbf{f}_{i}(\mathbf{s})\right) d \mathbf{f}_{i}(\mathbf{s})=\rho(\mathbf{s}) d \mathbf{s} .
$$

They also satisfy group properties [20], which ensure the indistinguishability of the $N$ electrons. The functional $V_{e e}^{\mathrm{SCE}}[\rho]$ is then given by

$$
V_{e e}^{\mathrm{SCE}}[\rho]=\int d \mathbf{s} \frac{\rho(\mathbf{s})}{N} \sum_{i=1}^{N} \sum_{j=i+1}^{N} \frac{1}{\left|\mathbf{f}_{i}(\mathbf{s})-\mathbf{f}_{j}(\mathbf{s})\right|} .
$$

Notice that, whereas, in chemistry, only the three-dimensional case is interesting, in physics, systems with reduced effective dimensionality (quantum dots, quantum wires, point contacts, etc.) play an important role.

As we see in Secs. IV and V, this way of addressing the functional $V_{e e}^{\mathrm{SCE}}[\rho]$ corresponds to an attempt to solve the so-called Monge problem associated with the constrained minimization of Eq. (6). In the Monge problem, one essentially tries to transport a mass distribution $\rho_{1}(\mathbf{r}) d \mathbf{r}$ into a mass distribution $\rho_{2}(\mathbf{r}) d \mathbf{r}$ in the most economical way according to a given definition of the work necessary to move a unit mass from position $\mathbf{r}_{1}$ to position $\mathbf{r}_{2}$. For example, one may wish to move books from one shelf ("shelf 1") to another ("shelf 2") by minimizing the total work. The goal of solving the Monge problem is then to find an optimal map, which assigns, to every book on shelf 1 , a unique final destination on shelf 2. In Secs. IV and V, it then becomes clear that the co-motion functions are the optimal maps of the Monge problem associated with $V_{e e}^{\mathrm{SCE}}[\rho]$.

However, it is well known in mass transportation theory that the Monge problem is very delicate and that proving, in general, the existence of the optimal maps (or co-motion functions) is extremely difficult. In 1942, Kantorovich proposed a relaxed formulation of the Monge problem in which the goal was now to find the probability that, when minimizing the total cost, a mass element of $\rho_{1}$ at position $\mathbf{r}_{1}$ be transported at position $\mathbf{r}_{2}$ in $\rho_{2}$. As detailed in Sec. V, this formulation is 
actually the appropriate one for the constrained minimization of Eq. (6).

We were then able to prove, in Sec. VI, four theorems on $V_{e e}^{\mathrm{SCE}}[\rho]$. In the first one, the existence of a generalized minimizer for Eq. (6) is rigorously established. It is useful to be reminded here that the functional $V_{e e}^{\mathrm{SCE}}[\rho]$ corresponds to the $\lambda \rightarrow \infty$ limit $[6,7]$ of the traditional adiabatic connection of DFT [22-25] in which a functional $F_{\lambda}[\rho]$, depending on a real parameter $\lambda$, is defined as

$$
F_{\lambda}[\rho]=\min _{\Psi \rightarrow \rho}\left\langle\Psi\left|\hat{T}+\lambda \hat{V}_{e e}\right| \Psi\right\rangle
$$

If $\Psi_{\lambda}[\rho]$ is the minimizer of Eq. (9), and if we define

$$
W_{\lambda}[\rho] \equiv\left\langle\Psi_{\lambda}[\rho]\left|\hat{V}_{e e}\right| \Psi_{\lambda}[\rho]\right\rangle-U[\rho]
$$

we have, under mild assumptions, the well-known exact formula [24] for the exchange-correlation functional of $\mathrm{KS}$ DFT,

$$
E_{x c}[\rho]=\int_{0}^{1} W_{\lambda}[\rho] d \lambda .
$$

When $\lambda \rightarrow \infty$, it can be shown that $[6,7,20,21]$

$$
\lim _{\lambda \rightarrow \infty} W_{\lambda}[\rho]=V_{e e}^{\mathrm{SCE}}[\rho]-U[\rho],
$$

where $U[\rho]$ is the Hartree functional of Eq. (5). We have, thus, set the existence of this limit, which contains a piece of exact information that can be used to model $E_{x c}[\rho][7,11-13,26,27]$ on firm ground.

When $\rho(\mathbf{r})$ is ground-state $v$-representable $\forall \lambda, \Psi_{\lambda}[\rho]$ is the ground state of the Hamiltonian,

$$
\hat{H}_{\lambda}[\rho]=\hat{T}+\lambda \hat{V}_{e e}+\hat{V}_{\lambda}[\rho],
$$

where

$$
\hat{V}_{\lambda}[\rho]=\sum_{i=1}^{N} v_{\lambda}[\rho]\left(\mathbf{r}_{i}\right)
$$

is a one-body local potential that keeps the density equal to the physical $(\lambda=1) \rho(\mathbf{r}) \forall \lambda$. In Refs. [9,20,21], it has been argued that

$$
\lim _{\lambda \rightarrow \infty} \frac{v_{\lambda}[\rho](\mathbf{r})}{\lambda}=v_{\mathrm{SCE}}[\rho](\mathbf{r}),
$$

where $v_{\mathrm{SCE}}[\rho](\mathbf{r})$ is related to the co-motion functions via the classical equilibrium equation [20],

$$
\nabla v_{\mathrm{SCE}}[\rho](\mathbf{r})=\sum_{i=2}^{N} \frac{\mathbf{r}-\mathbf{f}_{i}(\mathbf{r})}{\left|\mathbf{r}-\mathbf{f}_{i}(\mathbf{r})\right|^{3}},
$$

and it is the counterpart of the KS potential in the stronginteraction limit. In fact, we also have

$$
\frac{\delta V_{e e}^{\mathrm{SCE}}[\rho]}{\delta \rho(\mathbf{r})}=-v_{\mathrm{SCE}}[\rho](\mathbf{r}) \text {. }
$$

Although Eq. (16) is only valid if the co-motion functions (optimal maps) exist, Eq. (17) is more general. As we will see in Secs. V and VI, the Kantorovich problem can be rewritten in a useful dual formulation in which the so-called Kantorovich potential $u(\mathbf{r})$ plays a central role. The relation between the Kantorovich potential and $v_{\mathrm{SCE}}[\rho](\mathbf{r})$ is simply

$$
u(\mathbf{r})=-v_{\mathrm{SCE}}[\rho](\mathbf{r})+C,
$$

where $C$ is a constant that appears if we want to set $v_{\mathrm{SCE}}(|\mathbf{r}| \rightarrow$ $\infty)=0$ with $|\mathbf{r}|$ denoting the distance from the center of charge of the external potential. With our Theorems 2-4, we have proved that, under very mild assumptions on $\rho(\mathbf{r})$, this potential exists, it is bounded, and it is differentiable almost everywhere (a.e.) also for cases in which the co-motion functions do not exist, thus, addressing the $v$-representability problem in the strong interaction $(\lambda \rightarrow \infty)$ limit.

Theorem 4 also proves that the value of $V_{e e}^{\mathrm{SCE}}[\rho]$ is exactly given by the maximum of the Kantorovich dual problem,

$$
\begin{aligned}
& V_{e e}^{\mathrm{SCE}}[\rho] \\
& =\max _{u}\left\{\int u(\mathbf{r}) \rho(\mathbf{r}) d \mathbf{r}: \sum_{i=1}^{N} u\left(\mathbf{r}_{i}\right) \leqslant \sum_{i=1}^{N} \sum_{j>i}^{N} \frac{1}{\left|\mathbf{r}_{i}-\mathbf{r}_{j}\right|}\right\} .
\end{aligned}
$$

The condition $\sum_{i=1}^{N} u\left(\mathbf{r}_{i}\right) \leqslant \sum_{i=1}^{N} \sum_{j>i}^{N} \frac{1}{\left|\mathbf{r}_{i}-\mathbf{r}_{j}\right|}$ has a simple physical meaning: It requires that, at optimality, the allowed subspace $\Omega_{0}$ of the full $N d$ configuration space be a minimum of the classical potential energy. This can be easily verified by rewriting this condition in terms of $v_{\mathrm{SCE}}[\rho](\mathbf{r})$ using Eq. (18),

$$
\sum_{i=1}^{N} \sum_{j>i}^{N} \frac{1}{\left|\mathbf{r}_{i}-\mathbf{r}_{j}\right|}+\sum_{i=1}^{N} v_{\mathrm{SCE}}[\rho]\left(\mathbf{r}_{i}\right) \geqslant E_{\mathrm{SCE}}
$$

where the equality is satisfied only for configurations belonging to $\Omega_{0}$ and $E_{\mathrm{SCE}}$ is the total energy in the SCE limit [21]: $E_{\mathrm{SCE}}=\lim _{\lambda \rightarrow \infty} \lambda^{-1} E_{\lambda}$, where $E_{\lambda}$ is the ground-state energy of (13).

Equation (19) is related to the Legendre transform formulation of Lieb [28] of the KS functionals, but it has the advantage of being only a maximization under linear constraints, meaning that it can be dealt with linear programming techniques.

We were not able to prove the existence of the co-motion functions (optimal maps) in the general case, although we have hints that, for reasonable densities, it might be possible. As mentioned, this is always a delicate problem. We could only prove the existence of an optimal map in the special case $N=2$ (Appendix B).

In the following sections, we introduce the optimal transport problem, and we give the details of the results anticipated here.

\section{OPTIMAL TRANSPORT}

In 1781, Monge [3] proposed a model to describe the work necessary to move a mass distribution $p_{1}=\rho_{1} d x$ into a final destination $p_{2}=\rho_{2} d x$, given the unitary transportation cost function $c(x, y)$, which measured the work to move a unit mass from $x$ to $y$. The goal is to find a so-called optimal transportation map $f$, which moves $p_{1}$ into $p_{2}$, i.e., such that

$$
p_{2}(S)=p_{1}\left(f^{-1}(S)\right) \quad \forall \text { measurable sets } S,
$$

with minimal total transportation cost,

$$
\int c(x, f(x)) d p_{1} .
$$

The measures $p_{1}$ and $p_{2}$, which must have equal mass (normalized to one for simplicity), are called marginals. The natural framework for this kind of problem is the one 
where $X$ is a metric space and $p_{1}, p_{2}$ are probabilities on $X$. However, the existence of an optimal transport map is a very delicate question (for a simple example, see Sec. VII), even in the classical Monge case where $X$ is the Euclidean space $\mathbb{R}^{d}$ and the cost function is the distance between $x$ and $y, c(x, y)=|x-y|$. Thus, in 1942, Kantorovich [4] proposed a relaxed formulation of the Monge transport problem: The goal was now to find a probability $P(x, y)$ on the product space, which minimized the relaxed transportation cost,

$$
\int c(x, y) P(d x, d y)
$$

over all admissible probabilities $P$ where admissibility meant that the projections $\pi_{1}^{\#} P$ and $\pi_{2}^{\#} P$ coincided with the marginals $p_{1}$ and $p_{2}$, respectively. Here, the notation $\pi_{i}^{\#} P$ means that we integrate $P$ over all variables except the $i$ th. The Kantorovich problem then reads

$$
\min _{P}\left\{\int c(x, y) P(d x, d y): \pi_{j}^{\#} P=p_{j} \quad \text { for } j=1,2\right\},
$$

where $j=1,2$ denotes, respectively, the variables $x$ and $y$. The minimizing $P(d x, d y)=P(x, y) d x d y$ in Eq. (23), called the transport plan, gives the probability that a mass element in $x$ be transported in $y$ : This is evidently more general than the Monge transportation map $f$, which assigns a unique destination $y$ to each $x$.

The generalization to more than two marginals is crucial for our purpose and is written as

$$
\begin{gathered}
\min _{P}\left\{\int c\left(x_{1}, \ldots, x_{N}\right) P\left(d x_{1}, \ldots, d x_{N}\right):\right. \\
\left.\pi_{j}^{\#} P=p_{j} \quad \text { for } \quad j=1, \ldots, N\right\} .
\end{gathered}
$$

The analogous Monge problem in this case is to find $N$ maps $f_{i}$ such that $f_{1}(x)=x, p_{i}(S)=p_{1}\left(f_{i}^{-1}(S)\right)$ for every measurable set $S$, and $\left(f_{1}, \ldots, f_{N}\right)$ minimizes

$$
\int c\left(f_{1}\left(x_{1}\right), \ldots, f_{N}\left(x_{1}\right)\right) p_{1}\left(d x_{1}\right),
$$

among all maps with the same property.

\section{REFORMULATION OF $V_{e e}^{\mathrm{SCE}}[\rho]$}

We can now see that the way in which $V_{e e}^{\mathrm{SCE}}[\rho]$ was addressed in Refs. [20,21] (briefly reviewed in Sec. III) corresponds to an attempt to solve the Monge problem associated with the constrained minimization of Eq. (6) where the co-motion functions are the optimal maps. Indeed, Eq. (21) is a weak form of Eq. (7), which does not require $f$ to be differentiable.

However, as said, proving the existence of the optimal maps is, in general, a delicate problem. Moreover, the problem posed by Eq. (6) actually has the more general Kantorovich form (24). This can be seen by performing the following (with $x \in \mathbb{R}^{d}$ ):

(1) Identify the probability $P\left(d x_{1}, \ldots, d x_{N}\right)$ with $\left|\Psi\left(x_{1}, \ldots, x_{N}\right)\right|^{2} d x_{1}, \ldots, d x_{N}$;
(2) set all the marginals $p_{i}$ equal to the density divided by the number of particles $N, p_{i}=\frac{1}{N} \rho d x$;

(3) set the cost function equal to the electron-electron Coulomb repulsion,

$$
c\left(x_{1}, x_{2}, \ldots, x_{N}\right)=\sum_{i=1}^{N} \sum_{j=i+1}^{N} \frac{1}{\left|x_{i}-x_{j}\right|} .
$$

Thus, to solve the problem of finding the minimum possible electron-electron repulsion energy in a given density is equivalent to find the optimal way of transporting $N-1$ times the density $\rho$ into itself with the cost function given by the Coulomb repulsion in the relaxed Kantorovich formulation.

What are the advantages of this reformulation? As anticipated in Sec. III, we can set many of the conjectures on $V_{e e}^{\mathrm{SCE}}[\rho][9,11,20]$ on firm ground, and we can rewrite Eq. (6) in a convenient dual form that allows using linear programming techniques with the potential of giving access to a toolbox of algorithms already developed in a different well-established context.

\section{THEOREMS ON $V_{e e}^{\mathrm{SCE}}[\rho]$}

From the point of view of mass transportation theory, the problem of Eq. (6) poses two challenges: (i) The cost function corresponding to the Coulomb potential, Eq. (25), is different from the usual cost considered in the field. In particular, it is not bounded at the origin, and it decreases with distance, thus, requiring a generalized formal framework; (ii) the literature on the problem with several marginals is not very extensive (see, e.g., Refs. [29,30]). Nonetheless, we could prove several results. In what follows, we state them, relegating many technical details of the proofs to the Appendices.

Theorem 1. If the cost function $c$ is nonnegative and lower semicontinuous, there exists an optimal probability $P_{\text {opt }}$ for the minimum problem (24).

Proof. The proof is an application of the Prokhorov compactness theorem for measures. In fact, taken as a minimizing sequence $\left(P_{n}\right)$ for problem (24), since they are all probabilities, the sequence $\left(P_{n}\right)$ is compact in the weak* convergence of measures, so (a subsequence of) it converges weakly* to a non-negative measure $P$, and this is enough to obtain

$$
\int c d P \leqslant \liminf _{n} \int c d P_{n} .
$$

Then, $P$ is a good candidate for being an optimal probability for problem (24). To achieve the proof, it remains to show that $P$ is a probability and that the marginal condition $\pi_{j}^{\#} P=p_{j}$ is fulfilled. This is true if the convergence of $\left(P_{n}\right)$ to $P$ is "narrow," which, by Prokhorov theorem, amounts to show the so-called tightness condition,

$\forall \varepsilon>0 \exists K$ compact in $\mathbb{R}^{N d}: P_{n}\left(\mathbb{R}^{N d} \backslash K\right)<\varepsilon, \quad \forall n \in \mathbb{N}$.

The tightness condition above follows easily by the fact that all $P_{n}$ satisfy the marginal conditions $\pi_{j}^{\#} P_{n}=p_{j}(j=$ $1, \ldots, N)$.

Remark 1 . If the marginals $p_{1}, \ldots, p_{N}$ are all equal and if the cost function $c$ satisfies the symmetry condition,

$$
c\left(x_{1}, \ldots, x_{N}\right)=c\left(x_{k_{1}}, \ldots, x_{k_{N}}\right)
$$


for all permutations $k$, then the existence theorem above holds with $P_{\text {opt }}$, which satisfies the same symmetry condition. In fact, it is enough to notice that, taken as a probability $P$, the new probability,

$$
\tilde{P}\left(x_{1}, \ldots, x_{N}\right)=\frac{1}{N !} \sum_{k} P\left(x_{k_{1}}, \ldots, x_{k_{N}}\right)
$$

where $k$ runs over all permutations of $\{1, \ldots, N\}$, has a cost less than or equal to the one of $P$ and the same marginals.

We now turn to the important dual reformulation. The standard dual problem in optimal transport theory is as follows:

Theorem 2. Let $c$ be a lower semicontinuous and finitevalued function, then,

$$
\min _{P}\left\{\int c\left(x_{1}, \ldots, x_{N}\right) P\left(d x_{1}, \ldots, d x_{N}\right): \pi_{j}^{\#} P=p_{j} \text { for } j=1, \ldots, N\right\}=\max _{u_{j}}\left\{\sum_{j=1}^{N} \int u_{j} d p_{j}: \sum_{j=1}^{N} u_{j}\left(x_{j}\right) \leqslant c\left(x_{1}, \ldots, x_{N}\right)\right\} .
$$

Moreover, the dual maximization problem also admits a solution.

Remark 2. Again, if $p_{1}=\cdots=p_{N}=p$ and if the cost function $c$ satisfies the symmetry condition (26), then the dual problem admits a solution $u_{1}=\cdots=u_{N}=u$. In fact, if $u_{1}, \ldots, u_{N}$ is an optimal solution of the dual problem, the function,

$$
u(x)=\frac{1}{N}\left[u_{1}(x)+\cdots+u_{N}(x)\right]
$$

has the same maximal dual cost and satisfies the constraint,

$$
u\left(x_{1}\right)+\cdots+u\left(x_{N}\right) \leqslant c\left(x_{1}, \ldots, x_{N}\right) .
$$

Therefore, in this situation, the dual problem becomes

$$
\max _{u}\left\{N \int u d p: \sum_{i=1}^{N} u\left(x_{i}\right) \leqslant c\left(x_{1}, \ldots, x_{N}\right)\right\} .
$$

An optimal function $u$ for the dual problem (27) is called the Kantorovich potential.

However, the theorem above does not apply directly to the optimal transport problem of interest here because the cost, given by Eq. (25), takes the value $+\infty$ on the set $\left\{x_{i}=x_{j}\right.$ for some $\left.i \neq j\right\}$. The dual formulation then takes the following aspect (see, for instance, Ref. [31]).

Theorem 3. Let $c$ be a Borel function with values in $[0,+\infty]$, and assume that $c$ is $p_{1} \otimes \cdots \otimes p_{N}$ almost everywhere finite. Assume, moreover, that there exists a finite cost transport plan $\hat{P}$. Then, there exists Borel measurable dual maximizers $u_{i}$ with values in $[-\infty,+\infty)$ such that

$$
\begin{aligned}
& \min _{P}\left\{\int c\left(x_{1}, \ldots, x_{N}\right) P\left(d x_{1}, \ldots, d x_{N}\right): \pi_{j}^{\#} P=p_{j} \text { for } j=1, \ldots, N\right\} \\
& \quad=\max _{u_{j}}\left\{\int \sum_{j=1}^{N} u_{j}\left(x_{j}\right) d \hat{P}\left(d x_{1}, \ldots, d x_{N}\right): \sum_{j=1}^{N} u_{j}\left(x_{j}\right) \leqslant c\left(x_{1}, \ldots, x_{N}\right)\right\} .
\end{aligned}
$$

The assumption that $c$ is a Borel function is large enough to include continuous and lower semicontinuous functions (also taking the value $+\infty$ ), in particular, the Coulomb potential in Eq. (25).

The dual form of Theorem 3 does not allow explicit computations since it involves a plan $\hat{P}$, which may be not explicitly known. To overcome this difficulty, we were able to prove that, for the cost (25) under consideration, the more useful dual form (27) still holds:

Theorem 4. Let $c$ be the cost (25), and assume all marginal measures $p_{j}$ coincide. Then, there exists a maximizer $u$ for the dual problem of Theorem 3, which satisfies the formula,

$$
u(x)=\inf _{y_{i}}\left\{c\left(x, y_{1}, \ldots, y_{N-1}\right)-\sum_{i=1}^{N-1} u\left(y_{i}\right): y_{i} \in \mathbb{R}^{d}\right\}
$$

Such a Kantorovich potential $u$ is also bounded and verifies the equality,

$$
\int \sum_{j=1}^{N} u\left(x_{j}\right) d \hat{P}\left(d x_{1}, \ldots, d x_{N}\right)=N \int u(x) d p(x) .
$$

Moreover, if $p=\frac{1}{N} \rho(x) d x$, then $u$ is differentiable almost everywhere, and $\nabla u$ is locally bounded.

In Sec. III, we have already discussed the physical meaning of the Kantorovich potential $u$ : It is an effective single-particle potential, playing the same role of the KS potential in the strong-interaction limit.

The proof of Theorem 4 is discussed in Appendix A. We were also able to prove, as reported in Appendix $\mathrm{B}$, the existence of an optimal map (co-motion function) $f$ in the 
special case $N=2$, in any dimension $d$. In the following section, we show some explicit computations for simple cases.

\section{ANALYTICAL EXAMPLES}

The purpose of this section is to illustrate the optimal transport reformulation of the strictly correlated electron problem using simple examples. Results similar to those reported here have been already obtained from physical considerations in Refs. [6,11,18,20] where solutions using chemical and physical densities have been presented and have been discussed. In a way, this section is mainly addressed to the mass transportation community, with examples of the SCE problem translated in their familiar language. The DFT reader can also gain insight about the mass transportation formulation of the SCE problem from these examples by comparing them with those of Refs. [6,11,18,20].

We first consider the radial problem for two particles in a given dimension $d$ and then the case of $N$ particles in $d=1$ dimension.

\section{A. The radial $d$-dimensional case for $N=2$}

Here, we deal with the radial case $\rho(x)=\rho(|x|)$ when the number $N$ of particles is 2 .

The mass density $\rho(|x|)$ is transported on itself in an optimal way by a transport map $f$, whose existence has been proved in Appendix B. According to the one-dimensional calculations of the next subsection, for every half-line starting from the origin, the mass density $r^{d-1} \rho(r)$ is transported on the opposite half-line in an optimal way. In other words, we have

$$
f(x)=-\frac{x}{|x|} a(|x|),
$$

where the function $a(r)$ can be computed by solving the ordinary differential equation,

$$
a^{\prime}(r)[a(r)]^{d-1} \rho(a(r))=-r^{d-1} \rho(r),
$$

which gives

$$
\int_{0}^{a(r)} s^{d-1} \rho(s) d s=\frac{1}{d \omega_{d}}-\int_{0}^{r} s^{d-1} \rho(s) d s,
$$

where $\omega_{d}$ is the $d$ volume of the unit ball in $\mathbb{R}^{d}$. The Kantorovich potential $u(r)$ is obtained differentiating the dual relation $u(x)+u(y)=1 /|x-y|$ at the optimal points, which gives

$$
u(r)=-\int_{0}^{r} \frac{1}{[s+a(s)]^{2}} d s+\frac{1}{2} \int_{0}^{+\infty} \frac{1}{[s+a(s)]^{2}} d s .
$$

For instance, if $d=2$ and $\rho(r)$ is the Gaussian function $\rho(r)=$ $k e^{-k r^{2}} / \pi$, we find

$$
a(r)=\sqrt{-\frac{1}{k} \ln \left(1-e^{-k r^{2}}\right)} .
$$

Notice that these results were already obtained from physical arguments by Seidl [6] in his first paper on strictly correlated electrons.

It must also be noticed that replacing the Coulomb repulsion $1 /|x-y|$ by the more moderate repulsion (harmonic interaction) $-|x-y|^{2} / 2$, similar calculations give, due to the concavity of the cost function,

$$
f(x)=-x, \quad \text { with Kantorovich potential } u(r)=-r^{2},
$$

as already discussed in the Appendix of Ref. [20].

\section{B. The case $N=2$ and $d=1$ dimension}

We take $N=2$ particles in one dimension, and we first consider the simple case,

$$
\rho_{1}(x)=\rho_{2}(x)=\left\{\begin{array}{lc}
a, & \text { if }|x| \leqslant a / 2, \\
0, & \text { otherwise }
\end{array}\right.
$$

and

$$
c(x, y)=\frac{1}{|x-y|} .
$$

By symmetry, the goal is to send the interval $[0, a / 2]$ into $[-a / 2,0]$ by a transportation map $f$ with minimal cost,

$$
F(f)=a \int_{0}^{a / 2} \frac{1}{x-f(x)} d x .
$$

Since the function $t \mapsto 1 / t$ is convex on $\mathbb{R}^{+}$, by the Jensen inequality, we have

$$
F(f) \geqslant \frac{a^{3}}{4}\left(\int_{0}^{a / 2}(x-f(x)) d x\right)^{-1} .
$$

Taking into account that $\int_{0}^{a / 2} x d x=a^{2} / 8$ and

$$
\int_{0}^{a / 2} f(x) d x=\int_{-a / 2}^{0} y d y=-\frac{a^{2}}{8},
$$

we obtain that $F(f) \geqslant a$ for every transport map $f$. Choosing

$$
f(x)=x-\frac{a}{2},
$$

we have $F(f)=a$, which shows that $f$ is optimal. The plot of the optimal map $f$ on $[-a / 2, a / 2]$ is shown in Fig. 1. This is the same optimal map used in Ref. [18].

Similar computations can be performed for different densities $\rho$. Let us denote by $r_{1}$ the "first half" of $\rho$ and by $r_{2}$ the "second half;" there is no loss of generality if we assume that

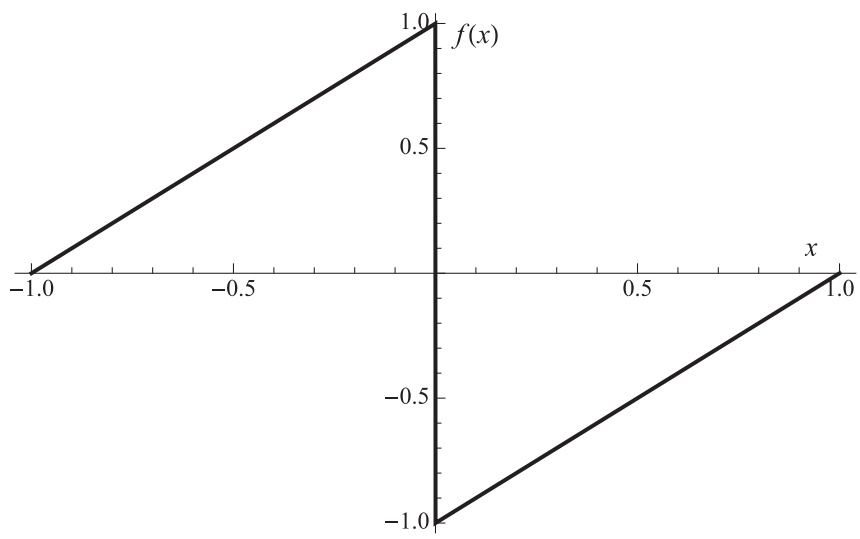

FIG. 1. The optimal map $f$ for the density of Eq. (28) with $a=2$. 


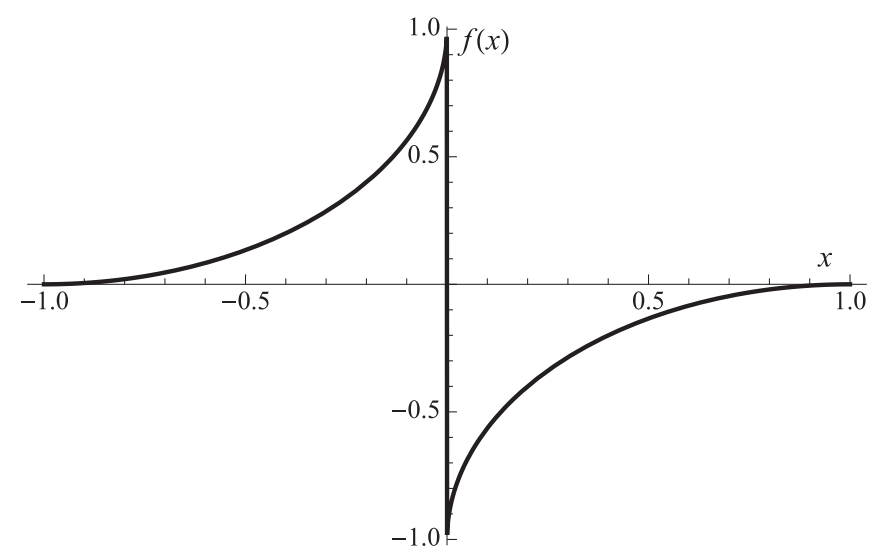

FIG. 2. The optimal map $f$ for the density of Eq. (29) in the case $a=1$.

the point where $\rho$ splits is the origin. In other words,

$$
\begin{aligned}
r_{1} & =\rho \text { on }]-\infty, 0\left[, \quad r_{2}=\rho \text { on }\right] 0,+\infty[, \quad \text { with } \\
\int_{-\infty}^{0} r_{1} d x & =\int_{0}^{+\infty} r_{2} d x=1 / 2 .
\end{aligned}
$$

The best transport map $f$ sends $r_{1}$ onto $r_{2}$, so from the differential relation,

$$
f^{\prime}(x) r_{2}(f(x))=r_{1}(x),
$$

taking into account that $f(-\infty)=0$, we find

$$
f(x)=R_{2}^{-1}\left(R_{1}(x)+\frac{1}{2}\right) \text { for } x<0,
$$

where $R_{1}$ and $R_{2}$ are the two primitives of $r_{1}$ and $r_{2}$, respectively, vanishing at the origin. Analogously, we obtain

$$
f(x)=R_{1}^{-1}\left(R_{2}(x)-\frac{1}{2}\right) \text { for } x>0,
$$

which agrees with the results of Refs. [6,20]. For instance, if

$$
\rho(x)=\frac{a-|x|}{a^{2}} \text { defined in }[-a, a],
$$

we get

$$
f(x)=\frac{x}{|x|}\left(\sqrt{2 a|x|-x^{2}}-a\right) \text { on }[-a, a]
$$

plotted in Fig. 2 for $a=1$.

Taking the Gaussian,

$$
\rho(x)=(\pi)^{-1 / 2} e^{-x^{2}},
$$

we obtain the optimal map shown in Fig. 3.

\section{The case $N \geqslant 3$ and $d=1$ dimension}

We consider the case of three particles in $\mathbb{R}$, with cost,

$$
c(x, y, z)=\frac{1}{|x-y|}+\frac{1}{|y-z|}+\frac{1}{|z-x|} .
$$

The transport maps formulation aims to find two maps $f_{1}: \mathbb{R} \rightarrow \mathbb{R}$ and $f_{2}: \mathbb{R} \rightarrow \mathbb{R}$ such that $f_{1}^{\#} \rho=f_{2}^{\#} \rho=\rho$, which minimize the quantity,

$$
\int_{\mathbb{R}}\left(\frac{1}{\left|x-f_{1}(x)\right|}+\frac{1}{\left|f_{1}(x)-f_{2}(x)\right|}+\frac{1}{\left|f_{2}(x)-x\right|}\right) d \rho(x),
$$

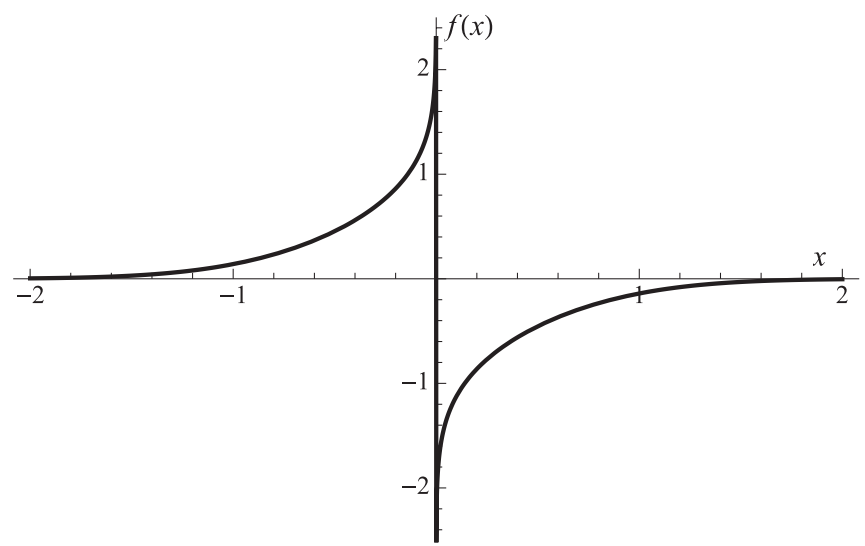

FIG. 3. The optimal map $f$ for the Gaussian density of Eq. (30).

with $f_{2}=f_{1} \circ f_{1}$ as it follows from the indistinguishability of the three particles.

The simplest case occurs when the marginal source $\rho$ is of the form,

$$
\rho=\frac{1}{3} \sum_{i=1}^{3} \delta_{x_{i}}
$$

in which the optimal transport maps $f_{i}$ are all the permutations of the points $\left\{x_{i}\right\}_{i=1,2,3}$ that do not send any point in itself. In the case of a diffuse source $\rho$, we split $\rho$ into its three tertiles $\rho_{1}, \rho_{2}, \rho_{3}$ with $\int \rho_{i} d x=1 / 3$, and we send $\rho_{1} \rightarrow \rho_{2}, \rho_{2} \rightarrow$ $\rho_{3}, \rho_{3} \rightarrow \rho_{1}$ through monotone transport maps. For instance, if $\rho$ is the Lebesgue measure on the interval $[0,1]$, we have that the optimal transport map $f_{1}$ is

$$
f_{1}(x)= \begin{cases}x+1 / 3, & \text { if } \quad x \leqslant 2 / 3 \\ x-2 / 3, & \text { if } \quad x>2 / 3\end{cases}
$$

and correspondingly,

$$
f_{2}(x)=f_{1}^{2}(x)=\left\{\begin{array}{lll}
x+2 / 3, & \text { if } & x \leqslant 1 / 3, \\
x-1 / 3, & \text { if } & x>1 / 3 .
\end{array}\right.
$$

The maps $f_{1}$ and $f_{2}$ are shown in Fig. 4. Let us show that $f_{1}$ and $f_{2}$ induce an optimal plan $P$. We check the optimality by calculating an explicit Kantorovich potential $u$, which satisfies, for all $x, y, z$,

$$
u(x)+u(y)+u(z) \leqslant \frac{1}{|x-y|}+\frac{1}{|x-z|}+\frac{1}{|y-z|},
$$

and, $\forall x$,

$$
\begin{aligned}
& u(x)+u\left(f_{1}(x)\right)+u\left(f_{2}(x)\right) \\
& =\frac{1}{\left|x-f_{1}(x)\right|}+\frac{1}{\left|x-f_{2}(x)\right|}+\frac{1}{\left|f_{1}(x)-f_{2}(x)\right|} .
\end{aligned}
$$

We remark that the right-hand side of Eq. (32) is equal to $15 / 2$. To calculate $u$, we observe that the inequality (31) holds everywhere, then, differentiating with respect to $x$, we obtain at a point $(x, y, z)$ of equality,

$$
u^{\prime}(x)=-\frac{x-y}{|x-y|^{3}}-\frac{x-z}{|x-z|^{3}} .
$$



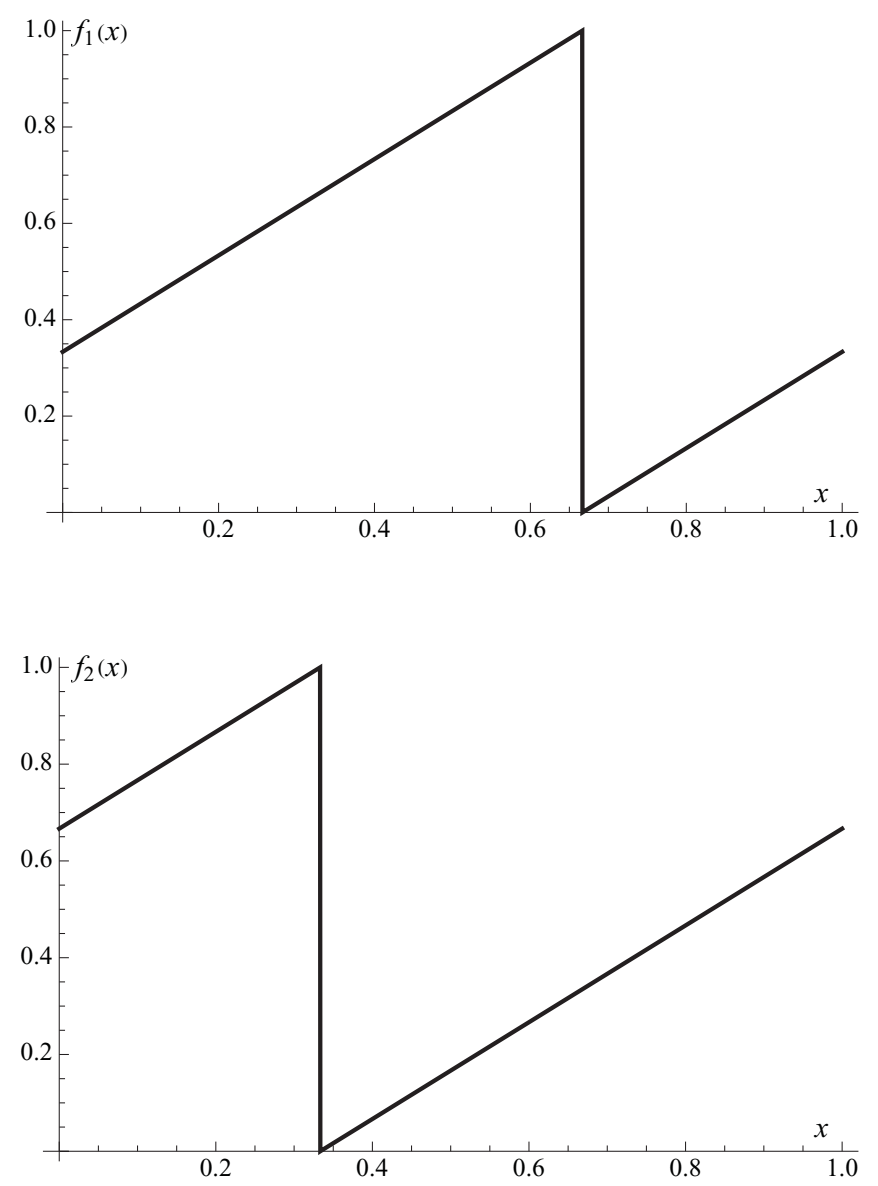

FIG. 4. The optimal maps $f_{1}$ and $f_{2}=f_{1}^{2}$ for $N=3$ and $\rho=d x$ on $[0,1]$.

Replacing $y$ by $f_{1}(x)$ and $z$ by $f_{2}(x)$, we obtain

$$
u^{\prime}(x)=\left\{\begin{array}{lll}
\frac{45}{4}, & \text { if } & x \in\left[0, \frac{1}{3}\right), \\
0, & \text { if } & x \in\left(\frac{1}{3}, \frac{2}{3}\right), \\
-\frac{45}{4}, & \text { if } & x \in\left(\frac{2}{3}, 1\right] .
\end{array}\right.
$$

Then, we find that

$$
u(x)= \begin{cases}\frac{45}{4} x+c, & \text { if } x \in\left[0, \frac{1}{3}\right], \\ \frac{15}{4}+c, & \text { if } x \in\left[\frac{1}{3}, \frac{2}{3}\right], \\ -\frac{45}{4} x+\frac{45}{4}+c, & \text { if } x \in\left[\frac{2}{3}, 1\right] .\end{cases}
$$

Equation (32) gives $c=0$. By construction, then, $u$ satisfies (32), and we only need to show that it also satisfies (31). To see this, we remark that, by symmetry, it is enough to check the inequality in the set where $x<y<z$ and that, on this set, the function,

$$
(x, y, z) \mapsto \frac{1}{|x-y|}+\frac{1}{|x-z|}+\frac{1}{|y-z|}
$$

is convex. On the other hand, by the concavity of $u$, the function,

$$
(x, y, z) \mapsto u(x)+u(y)+u(z)
$$

is concave. These two maps coincide together with their gradients at the point $(1 / 6,3 / 6,5 / 6)$, and then the convex one has to stay above the concave one.
In the case of a possibly singular source, optimal maps do not, in general, exist, and the optimal configurations are given by probability plans $P$. For instance, if

$$
\rho=\frac{1}{4} \sum_{i=1}^{4} \delta_{x_{i}}
$$

with $x_{i}$ ordered in an increasing way, we have that the optimal transport plan sends

$$
\begin{aligned}
& \delta_{x_{1}}+\frac{1}{3} \delta_{x_{2}} \rightarrow \frac{2}{3} \delta_{x_{2}}+\frac{2}{3} \delta_{x_{3}}, \\
& \frac{2}{3} \delta_{x_{2}}+\frac{2}{3} \delta_{x_{3}} \rightarrow \frac{1}{3} \delta_{x_{3}}+\delta_{x_{4}}, \\
& \frac{1}{3} \delta_{x_{3}}+\delta_{x_{4}} \rightarrow \delta_{x_{1}}+\frac{1}{3} \delta_{x_{2}} .
\end{aligned}
$$

When $N \geqslant 4$, similar arguments as above can be developed, giving transport maps $f_{1}, f_{1}^{2}, \ldots, f_{1}^{N-1}$ that minimize the total cost,

$$
\int c\left(x, f_{1}(x), f_{1}^{2}(x), \ldots, f_{1}^{N-1}(x)\right) d \rho(x),
$$

where $c\left(x_{1}, \ldots, x_{N}\right)$ is given in Eq. (25). Some of these results were also obtained by Seidl [6], again using physical arguments.

\section{CONCLUSIONS AND PERSPECTIVES}

We have shown that the strong-interaction limit of electronic density-functional theory can be rewritten as a mass transportation theory problem, thus, creating a link between two different well-established research areas. This is already interesting per se: It allows importing and generalizing results from one domain to the other. In particular, with our reformulation, we were able to prove immediately several results on the strong-interaction limit of DFT. Even more interesting, we could show that the problem of finding the minimum interaction energy in a given density can be rewritten in a convenient dual form consisting of a minimization under linear constraints, paving the way for the use of linear programming techniques to solve the strictly correlated electron problem.

Dual reformulations have already proved very useful in the context of electronic structure calculations: for example, in Ref. [32], the solution of the physical Hamiltonian by optimizing the second-order reduced density matrix has been tackled with a suitable dual problem. The use of Legendre transform techniques for the simplification of minimizations involving permutations in the many-electron problem has also been stressed and has been applied in Refs. [33,34] with very interesting results. All these approaches focused on the quantum-mechanical problem, whereas, here, we deal with a special problem that is essentially of classical nature but contains quantum-mechanical information via the electronic density. We know now that the optimal transport formulation is the right mathematical framework for the strong-interaction limit of density-functional theory.

It is also worth mentioning that the formalism developed here can be of interest for approaches to the many-electron problem, which use $k$-electron distribution functions (i.e., the diagonal of the $k$ th-order reduced density matrix), such as those of Refs. [35,36]. In fact, in these approaches, one usually constructs a $k$-electron distribution function $\rho_{k}\left(\mathbf{r}_{1}, \ldots, \mathbf{r}_{k}\right)$ 
with a given density, possibly minimizing the electron-electron repulsion energy. This would result in the same Kantorovich formulation considered here.

The formal and practical aspects of our new reformulation are enticing for DFT: making routinely available the piece of exact information, contained in the strong-interaction limit, can largely broaden its applicability, both by developing a SCE DFT [9-11] (which uses a strong-interacting system as a reference) and via new exchange-correlation functionals for standard KS DFT [8,11,13]. Future work will be devoted to exploit the practical aspects of this reformulation.

Note added. Recently, we became aware that a related paper [37] was posted on the arXiv. In Ref. [37], the particle-particle interaction term is minimized by only considering the pair density. By neglecting the $N$-representability issue, this leads to a two-particle problem with only one map (or co-motion function).

\section{ACKNOWLEDGMENTS}

This work was supported by the Netherlands Organization for Scientific Research (NWO) through a Vidi grant. P.G.-G. thanks R. Assaraf for suggesting reading about mass transportation theory, G. Vignale and M. Seidl for useful discussions, and A. Mirtschink for a critical reading of the manuscript.

\section{APPENDIX A: PROOF OF THEOREM 4}

For the sake of simplicity, we give a sketch of the proof only in the case of two particles; the general case can be obtained in a similar way. We, thus, consider the case $N=2$ in $\mathbb{R}^{d}$ with two equal marginals, $p_{1}=p_{2}=p$ with $p \in P\left(\mathbb{R}^{d}\right)$. The problem is then

$$
\min \left\{\int \frac{1}{|x-y|} d P(x, y): \pi_{j}^{\#} P=p_{j} \text { for } j=1,2\right\},
$$

and $p$ is assumed absolutely continuous, i.e., of the form $\rho(x) d x$ with $0 \leqslant \rho(x)$ and $\int \rho(x) d x=1$.

By definition, the Kantorovich potential $u$ is a maximizer for the dual problem according to Theorem 3 and Remark 1. If we denote by $P$ an optimal plan of transport (which exists by Theorem 1 ), then, in the case $N=2$ considered here, $u$ maximizes the functional,

$$
\int_{\mathbb{R}^{d} \times \mathbb{R}^{d}}(u(x)+u(y)) d P(x, y),
$$

among all the functions, which satisfy the constraint,

$$
u(x)+u(y) \leqslant \frac{1}{|x-y|} .
$$

Under the current assumptions, such a maximizer exists by Theorem 3 above, but $u$ is only a Borel function, which takes values in $[-\infty,+\infty)$. Much more then is needed to carry on the necessary computations, and we deduce the needed properties. The proof will be performed in several steps. But first, let us fix the following notation: For a transport plan $P$, we denote by $\operatorname{spt}(P)$ the support of $P$, i.e., the smallest closed set $F$ such that $P\left(\mathbb{R}^{d} \times \mathbb{R}^{d} \backslash F\right)=0$.
Step 1. The first step is the following intuitive fact about optimal transport plans. If $P_{\text {opt }}$ is an optimal transport plan, then

$$
0<|x-y| \quad \forall(x, y) \in \operatorname{spt}\left(P_{\text {opt }}\right) .
$$

Indeed, if, by contradiction, a point $(\bar{x}, \bar{x}) \in \operatorname{spt}\left(P_{\text {opt }}\right)$, we may find a better transport plan $\tilde{P}$ by exchanging the mass around $(\bar{x}, \bar{x})$ with the one around another point $(\tilde{x}, \tilde{y}) \in \operatorname{spt}\left(P_{\text {opt }}\right)$ having $\tilde{x} \neq \tilde{y} \neq \bar{x}$.

Step 2. Actually, something more can be said. Let $P_{\text {opt }}$ be an optimal transport plan; then, for all $R>0$, there exists $\alpha(R)>0$ such that

$$
\alpha(R)<|x-y| \quad \forall x \in B(0, R),
$$

where we denote by $B(x, r)$ the ball centred at $x$ and of radius $r$. Indeed, let $x \in B(0, R)$ and $(x, y) \in \operatorname{spt}\left(P_{\text {opt }}\right)$; by the point above and by compactness, the diagonal and the support of $P_{\text {opt }}$ have positive distances in the set $\overline{B(0, R)} \times \overline{B(0,2 R)}$, and we denote such a distance by $\beta(R)$. It follows that

$$
\min \{\beta(R), R\} \leqslant|x-y| \text {. }
$$

We then define $\alpha(R):=\min \{\beta(R), R\}$. Moreover, we may choose the function $\alpha$ nonincreasing.

Step 3. Using the second step, we now prove that there are Kantorovich potentials, which are bounded. First, we remark that we can choose a Kantorovich potential $v$, which satisfies

$$
v(x)=\inf _{y \in \mathbb{R}^{d}}\left\{\frac{1}{|x-y|}-v(y)\right\} .
$$

We start with a potential $u$, and we notice that, by definition,

$$
u(x) \leqslant \inf _{y \in \mathbb{R}^{d}}\left\{\frac{1}{|x-y|}-u(y)\right\} .
$$

Then, we can consider

$$
\bar{u}(x)=\inf _{y \in \mathbb{R}^{d}}\left\{\frac{1}{|x-y|}-u(y)\right\} .
$$

Clearly, $u \leqslant \bar{u}$. Even if $\bar{u}$ does not satisfy the constraint, from the definition, we get

$$
\begin{aligned}
\bar{u}(x)+\bar{u}(y)= & \inf _{z \in \mathbb{R}^{d}}\left\{\frac{1}{|x-z|}-u(z)\right\} \\
& +\inf _{z \in \mathbb{R}^{d}}\left\{\frac{1}{|z-y|}-u(z)\right\},
\end{aligned}
$$

and taking $y$ as a test in the first term on the right-hand-side and $x$ in the second, it follows that:

$$
\bar{u}(x)+\bar{u}(y) \leqslant \frac{2}{|x-y|}-u(y)-u(x),
$$

or equivalently, if we define $\tilde{u}(x)=2^{-1}(u(x)+\bar{u}(x))$,

$$
u(x) \leqslant \tilde{u}(x) \leqslant \bar{u}(x) \text { and } \tilde{u}(x)+\tilde{u}(y) \leqslant \frac{1}{|x-y|} .
$$

We may now define

$v(x)=\sup \{w(x): u(x) \leqslant w(x) \leqslant \bar{u}(x)$ and $w$ satisfies $(A 3)\}$.

The function $v(x)$ clearly satisfies (A3), and if $v \neq \bar{v}$, since

$$
\begin{aligned}
\bar{v}(x) & =\inf _{y \in \mathbb{R}^{d}}\left\{\frac{1}{|x-y|}-v(y)\right\} \leqslant \inf _{y \in \mathbb{R}^{d}}\left\{\frac{1}{|x-y|}-u(y)\right\} \\
& =\bar{u}(x),
\end{aligned}
$$


then, $v<\tilde{v} \leqslant \bar{u}$, which contradicts the maximality of $v$. Finally, $v$ maximizes the cost (A2) since $u \leqslant v$.

Step 4. As anticipated in Theorem 4, if $v$ is a Kantorovich potential which satisfies (A4), then there exists a constant $C$ such that $|v| \leqslant C$. Let $P_{\text {opt }}$ be an optimal plan of transport. The condition,

$$
\int_{\mathbb{R}^{d} \times \mathbb{R}^{d}}(v(x)+v(y)) d P_{\mathrm{opt}}(x, y)=\int_{\mathbb{R}^{d} \times \mathbb{R}^{d}} \frac{d P_{\mathrm{opt}}(x, y)}{|x-y|},
$$

together with the condition,

$$
v(x)+v(y) \leqslant \frac{1}{|x-y|}
$$

implies that

$$
v(x)+v(y)=\frac{1}{|x-y|} \text { for } P_{\mathrm{opt}} \text {-a.e. } x \text { and } y,
$$

and then, in particular, $v$ is finite $\rho$-a.e. Moreover, setting

$$
\begin{aligned}
G & =\{x:-\infty<v(x) \text { and } \exists y \text { such that } v(x)+v(y) \\
& \left.=\frac{1}{|x-y|}\right\}
\end{aligned}
$$

it follows from the discussion above that $\rho(G)=1$. Let $\bar{x} \in G$ be a point of density 1 for $G$, and let $\alpha$ and $r$ be such that $\alpha>r$ and

(1) for all $s \leqslant r$, we have $|B(\bar{x}, s) \cap G| /|B(\bar{x}, s)| \geqslant 3 / 4$,

(2) for all $x \in B(\bar{x}, r)$, if $(x, y) \in \operatorname{spt}(P)$, then $\alpha<|x-y|$.

Setting $L=v(\bar{x})$, we have that, for every $z \in \mathbb{R}^{d} \backslash$ $B(\bar{x}, r / 4)$,

$$
v(z)=\inf _{y \in \mathbb{R}^{d}}\left\{\frac{1}{|y-z|}-v(y)\right\} \leqslant \frac{4}{r}-L .
$$

On the other hand, for every $z \in B(\bar{x}, r / 4) \cap G$, there exists $y$ such that $\alpha<|z-y|,(z, y) \in \operatorname{spt}\left(P_{\text {opt }}\right)$, and $v(z)+v(y)=$ $1 /|z-y|$. Then,

$$
\begin{aligned}
v(z) & =\inf _{y \in \mathbb{R}^{d}}\left\{\frac{1}{|y-z|}-v(y)\right\} \leqslant \inf _{|y-z| \geqslant \alpha}\left\{\frac{1}{|y-z|}-v(y)\right\} \\
& =v(z),
\end{aligned}
$$

since $r<\alpha, \mathbb{R}^{d} \backslash B(z, \alpha) \subset \mathbb{R}^{d} \backslash B(\bar{x}, r / 4)$, and then, from the estimate (A5),

$$
v(z)=\inf _{|y-z| \geqslant \alpha}\left\{\frac{1}{|y-z|}-v(y)\right\} \geqslant L-\frac{1}{r} .
$$

To get control of $v$ from above in $B(\bar{x}, r / 4)$, we observe that, if $\lambda \leqslant r 4^{-1 / d}$ and $z \in B(\bar{x}, r / 4)$, since

$$
|B(z, \lambda)|=\omega_{d} \lambda^{d} \leqslant \frac{\omega_{d}}{4} r^{d},
$$

then, there exists at least one $y_{z} \in G \cap B(\bar{x}, r / 4) \backslash B(z, \lambda)$ such that, from the estimate (A6),

$$
\begin{aligned}
v(z) & \leqslant \inf _{|y-z| \geqslant \lambda}\left\{\frac{1}{|y-z|}-v(y)\right\} \leqslant \frac{1}{\left|y_{z}-z\right|}-v\left(y_{z}\right) \\
& \leqslant \frac{1}{\lambda}+\frac{1}{r}-L .
\end{aligned}
$$

Estimates (A5) and (A7) give a bound from above on $v$ by a constant $K$. The estimate from below is now straightforward since

$$
v(x)=\inf _{\mathbb{R}^{d}}\left\{\frac{1}{|x-y|}-v(y)\right\} \geqslant-K .
$$

Step 5. The previous steps permit gaining more regularity on the potential $v$. Indeed, let $v$ be a Kantorovich potential, which satisfies (A4); we show that $v$ is differentiable almost everywhere. To see this, we consider the family of functions,

$$
v_{n}(x)=\inf _{\alpha(n)<|x-y|}\left\{\frac{1}{|x-y|}-v(y)\right\} .
$$

Since $\alpha$ is nonincreasing, we have

$$
v_{n+1}(x) \leqslant v_{n}(x)
$$

Moreover, each $v_{n}$ is a Lipschitz function of Lipschitz constant $1 / \alpha^{2}(n)$. By Step 2, for $x \in G$, if $|x|<m<n$, then $v(x)=v_{n}(x)=v_{m}(x)$. Then, on $G$, the potential $v$ coincides locally with a Lipschitz function, which is well known to be differentiable almost everywhere.

\section{APPENDIX B: PROOF OF THE EXISTENCE OF AN OPTIMAL TRANSPORT MAP FOR $N=2$}

Once the existence of an almost everywhere differentiable Kantorovich potential $v$ is established, we may consider the problem of showing the existence of an optimal transport map (co-motion function) $f$. In the case $N=2$, the proof can be achieved by using the basic idea of differentiating inequality (A3) at the points of equality.

Let $P_{\text {opt }}$ be an optimal transport plan, and let $G$ be defined as above. If $G_{N}:=G \cap B(0, N)$, we prove that, for almost every $x \in G_{N}$, there exists only one $y$ such that $(x, y) \in \operatorname{spt}\left(P_{\text {opt }}\right)$, and we give an explicit expression for such $y$. It follows that $P_{\text {opt }}$ is induced by an optimal transport map. Let $v_{N}$ be the function defined above since $v_{N}$ coincides with $v$ on $G_{N}$, for every $x \in G_{N}$ and $y$, the inequality

$$
v_{N}(x)+v(y) \leqslant \frac{1}{|x-y|}
$$

holds. Since $P_{\mathrm{opt}}$ is an optimal transport plan and $\rho=a(x) d x$, then, for $P_{\text {opt }}$-a.e. $(x, y) \in \operatorname{spt}\left(P_{\text {opt }}\right), x$ belongs to $G_{N}$ for a suitable $N, x$ is a density point for $G_{N}$, and $v_{N}$ is differentiable at $x$. Since for $z \in G_{N}$,

$$
v_{N}(z) \leqslant \frac{1}{|z-y|}-v(y),
$$

and equality holds for $z=x$, then, if we differentiate the functions $v_{N}$ and $\psi(z)=\frac{1}{|z-y|}-v(y)$, we obtain

$$
\nabla v_{N}(x)=-\frac{x-y}{|x-y|^{3}},
$$

from which, it follows:

$$
y=x+\frac{1}{\left|\nabla v_{N}(x)\right|^{3 / 2}} \nabla v_{N}(x) .
$$

From Eq. (B1), we deduce that, for $P_{\text {opt }}$-a.e. $(x, y)$, point $y$ is uniquely determined by $x$, and this concludes the proof of the existence of an optimal transport map $f$, by defining

$$
f(x)=x+\frac{1}{\left|\nabla v_{N}(x)\right|^{3 / 2}} \nabla v_{N}(x),
$$

whenever $x \in B(0, N)$. 
[1] P. Hohenberg and W. Kohn, Phys. Rev. 136, B864 (1964).

[2] W. Kohn and L. J. Sham, Phys. Rev. 140, A1133 (1965).

[3] G. Monge, Mémoire sur la Théorie des Déblais et des Remblais (Histoire Acad. Sciences, Paris, 1781).

[4] L. V. Kantorovich, Dokl. Akad. Nauk SSSR 37, 227 (1942).

[5] M. Levy, Proc. Natl. Acad. Sci. USA 76, 6062 (1979).

[6] M. Seidl, Phys. Rev. A 60, 4387 (1999).

[7] M. Seidl, J. P. Perdew, and M. Levy, Phys. Rev. A 59, 51 (1999).

[8] M. Seidl, J. P. Perdew, and S. Kurth, Phys. Rev. Lett. 84, 5070 (2000).

[9] P. Gori-Giorgi, M. Seidl, and G. Vignale, Phys. Rev. Lett. 103, 166402 (2009).

[10] Z. F. Liu and K. Burke, J. Chem. Phys. 131, 124124 (2009).

[11] P. Gori-Giorgi and M. Seidl, PhysChemChemPhys 12, 14405 (2010).

[12] A. M. Teale, S. Coriani, and T. Helgaker, J. Chem. Phys. 132, 164115 (2010)

[13] A. Becke, Abstr. Pap. - Am. Chem. Soc. 242 (2011).

[14] E. H. Lieb, Phys. Lett. A 70, 444 (1979).

[15] E. H. Lieb and S. Oxford, Int. J. Quantum. Chem. 19, 427 (1981).

[16] J. P. Perdew, in Electronic Structure of Solids '91, edited by P. Ziesche and H. Eschrig (Akademie Verlag, Berlin, 1991).

[17] M. Levy and J. P. Perdew, Phys. Rev. B 48, 11638 (1993).

[18] E. Räsänen, M. Seidl, and P. Gori-Giorgi, Phys. Rev. B 83, 195111 (2011).

[19] M. Seidl, J. P. Perdew, and S. Kurth, Phys. Rev. A 62, 012502 (2000).
[20] M. Seidl, P. Gori-Giorgi, and A. Savin, Phys. Rev. A 75, 042511 (2007).

[21] P. Gori-Giorgi, G. Vignale, and M. Seidl, J. Chem. Theory Comput. 5, 743 (2009).

[22] J. Harris and R. Jones, J. Phys. F 4, 1170 (1974).

[23] J. Harris, Phys. Rev. A 29, 1648 (1984).

[24] D. C. Langreth and J. P. Perdew, Solid State Commun. 17, 1425 (1975).

[25] M. Levy, Phys. Rev. A 43, 4637 (1991).

[26] M. Ernzerhof, Chem. Phys. Lett. 263, 499 (1996).

[27] K. Burke, M. Ernzerhof, and J. P. Perdew, Chem. Phys. Lett. 265, 115 (1997).

[28] E. H. Lieb, Int. J. Quantum Chem. 24, 243 (1983).

[29] W. Gangbo and A. Swiech, Commun. Pure Appl. Math. 51, 23 (1998).

[30] S. Rachev and L. Rüschendorf, Mass Transportation Problems (Springer-Verlag, New York, 1998).

[31] M. Beiglboeck, C. Léonard, and W. Schachermayer, arXiv:0911.4347.

[32] E. Cancès, G. Stoltz, and M. Lewin, J. Chem. Phys. 125, 064101 (2006).

[33] J. E. Osburn and M. Levy, Phys. Rev. A 33, 2230 (1986).

[34] J. E. Osburn and M. Levy, Phys. Rev. A 35, 3233 (1987).

[35] P. W. Ayers, Phys. Rev. A 74, 042502 (2006).

[36] B. Liu, Ph.D. thesis, New York University, 2006.

[37] C. Cotar, G. Friesecke, and C. Klüppelberg, arXiv:1104.0603. 